\title{
Politiques patrimoniales et réhabilitation urbaine au Portugal
} Mr Carlos Fortuna, Monsieur Paulo Peixoto

\section{Citer ce document / Cite this document :}

Fortuna Carlos, Peixoto Paulo. Politiques patrimoniales et réhabilitation urbaine au Portugal. In: Pôle Sud, n²2, 2005. pp. 127141 ;

doi : 10.3406/pole.2005.1225

http://www.persee.fr/doc/pole_1262-1676_2005_num_22_1_1225

Document généré le 10/06/2016 


\begin{abstract}
The analysis of urban planning and of the rehabilitation of Portugal's architectural and urban historical heritage over the past fifteen years demonstrates a clear intensification of local processes aimed at preserving and enhancing historical sites. Three trends in particular are evident. The first is the effort to gain official recognition of world heritage sites. In addition, we note the spread of international methods of urban management. Finally there is a tendency towards excessive "packaging" or esthetic presentation of public spaces. Taking as examples the historic centers of four Portuguese cities, (Coimbra, Guimarâes, Porto, and Santarém) this article seeks to analyze the specificities of these policies with a view to understanding their experimental nature and to assessing the cultural sustainability as well of the policies themselves as of the urban centers that are their objects.
\end{abstract}

\title{
Résumé
}

L'analyse des politiques patrimoniales et des opérations de réhabilitation urbaine au Portugal fait ressortir, dans les quinze dernières années, une nette intensification des processus locaux de patrimonialisation. Cette intensification est marquée par trois processus : par une course au titre de patrimoine mondial, par l'importation des modèles mondialisés de gestion urbaine et par des formes exacerbées d'animation et d'esthétisation de l'espace. En prenant des exemples concernant les centres historiques de quatre villes portugaises (Coimbra, Guimarâes, Porto et Santarém), nous essayons de mettre en évidence les spécificités des politiques patrimoniales et des opérations de réhabilitation urbaine au Portugal, afin de rendre compte du caractère expérimental qui les façonne et de questionner la "sou- tenabilité » culturelle de ces politiques et des centres urbains où elles sont adoptées. 


\title{
Politiques patrimoniales et réhabilitation urbaine au Portugal
}

\author{
Carlos Fortuna et Paulo Peixoto \\ Centre d'Études Sociales, Université de Coimbra
}

\section{Résumé/Abstract}

L'analyse des politiques patrimoniales et des opérations de réhabilitation urbaine au Portugal fait ressortir, dans les quinze dernières années, une nette intensification des processus locaux de patrimonialisation. Cette intensification est marquée par trois processus: par une course au titre de patrimoine mondial, par l'importation des modèles mondialisés de gestion urbaine et par des formes exacerbées d'animation et d'esthétisation de l'espace. En prenant des exemples concernant les centres historiques de quatre villes portugaises (Coimbra, Guimarães, Porto et Santarém), nous essayons de mettre en évidence les spécificités des politiques patrimoniales et des opérations de réhabilitation urbaine au Portugal, afin de rendre compte du caractère expérimental qui les façonne et de questionner la " soutenabilité " culturelle de ces politiques et des centres urbains où elles sont adoptées.

The analysis of urban planning and of the rehabilitation of Portugal's architectural and urban historical heritage over the past fifteen years demonstrates a clear intensification of local processes aimed at preserving and enhancing historical sites. Three trends in particular are evident. The first is the effort to gain official recognition of world heritage sites. In addition, we note the spread of international methods of urban management. Finally there is a tendency towards excessive "packaging" or esthetic presentation of public spaces. Taking as examples the historic centers of four Portuguese cities, (Coimbra, Guimaräes, Porto, and Santarem) this article seeks to analyze the specificities of these policies with a view to understanding their experimental nature and to assessing the cultural sustainability as well of the policies themselves as of the urban centers that are their objects.

\section{$\approx$ \\ Mots-Clés/Keywords}

Patrimoine mondial, politiques patrimoniales, réhabilitation urbaine, soutenabilité culturelle, tourisme et loisirs urbains

Heritage policies, urban rehabilitation, urban sustainability, urban tourism and leisure, world heritage 
La restauration et l'animation de centres historiques, de zones monumentales et de places publiques constituent une dimension importante des nouvelles formes architectoniques et urbanistiques. Celles-ci, prises dans le cadre de la compétitivité à laquelle se livrent les villes - et les États -, reconnaissent dans la culture, dans l'esprit du lieu, et dans le patrimoine urbain un terrain à partir duquel peut se créer et s'inventer une différence que les villes ont à offrir dans le contexte des flux d'un tourisme historique et patrimonial, de la consolidation d'un marché urbain de loisirs ou des stratégies d'attraction de nouveaux résidents et investisseurs. Bref, le patrimoine et la rhétorique patrimoniale répondent très bien aux exigences des idéologies urbaines actuelles: qualité de vie, développement durable, tourisme non massifié, récupération de l'espace public, participation civique, etc.

Cette dynamique patrimoniale est le résultat de l'accroissement et de l'amplification de l'esprit de concurrence entre différents espaces pour lesquels le statut de patrimoine mondial se présente comme un faire-valoir dans trois domaines: en tant que vecteur des stratégies de marketing urbain; en tant qu'atout qui cherche à attirer les flux du tourisme culturel et patrimonial; et comme un élément rhétorique de légitimation et de contestation des politiques de transformation des paysages urbains. À la limite, cette dynamique participe d'une reproduction en série des modèles mondialisés de développement urbain, particulièrement conçus pour être compétitifs sur le marché touristique et pour produire de l'exemplarité et de la fascination au niveau des opérations de réhabilitation. Ces modèles, comme nous le constatons au Portugal, sont appliqués tout en faisant ressortir un évident caractère expérimental.

Lintensification des processus de patrimonialisation, surtout celle motivée par une course au statut de patrimoine mondial ainsi que les règles, récemment adoptées par l'Unesco pour combattre cette intensification, sont analysées dans ce texte à partir de quatre idées principales. D'abord, nous cherchons à démontrer et à caractériser brièvement la course au statut de patrimoine mondial en soulignant les conflits nationaux et locaux qu'elle finit par faire émerger. Ensuite, nous plaçons les jalons qui permettent de mettre en évidence les rapports entre le patrimoine, et notamment le statut de patrimoine mondial, et le marketing urbain. Après, nous nous interrogeons sur la nature des relations entre politiques de patrimonialisation, tourisme et 
promotion d'un marché urbain des loisirs. Enfin, nous cernons les politiques locales patrimoniales et les opérations de réhabilitation dans le domaine représentationnel et symbolique pour questionner leur « soutenabilité " culturelle ${ }^{1}$. Des cas sélectionnés, portant sur des politiques patrimoniales conduites à Coimbra, Guimarães, Porto et Santarém, nous permettront d'exemplifier et d'exploiter ces idées.

\section{La course au titre de patrimoine mondial}

Au niveau mondial, l'une des caractéristiques de la dernière décennie du millénaire est l'intensification des processus de patrimonialisation. Si l'on se réfere à la figure emblématique du patrimoine mondial, instituée par la Convention de l'Unesco en 1972, nous constatons que, durant les années 1990, a émergé une sorte de course dont le but est l'acquisition du statut de patrimoine mondial (Peixoto, 2002a). Au Portugal, dès qu'en 1983 l'Unesco a octroyé cette distinction au centre historique d'Angra do Heroismo (situé aux Açores), au Monastère des Hiéronymites et à la Tour de Belém, à Lisbonne, au Monastère de Batalha et au Couvent du Christ, à Tomar, nous avons assisté à une multiplication de candidatures, effectives ou seulement envisagées, au statut de patrimoine mondial. Dans les années 1980 , cette reconnaissance a été obtenue par le centre historique d'Évora, en 1986, et le Monastère d'Alcobaça, en 1989. Durant les années 1990, plusieurs villes et biens ont été distinguées: le paysage culturel de Sintra, en 1995, le centre historique de Porto, en 1996, les Sites d'Art Rupestre Préhistorique de la Vallée de Côa, en 1998, et la Forêt Laurifière de Madère, en 1999. À ces sites s'ajoute, depuis 2001, la région viticole du " Haut Douro » et le centre historique de
Guimarães et, depuis 2004, le Paysage viticole de l'île du Pico (aux Açores). Outre ces 13 biens du patrimoine mondial, une vingtaine ${ }^{2}$ d'autres biens font partie ou souhaitent faire partie de la liste préalable que l'État portugais, en référence à la Convention de 1972, doit remettre à l'Unesco tous les 5 ans.

Comme nous avons pu le constater par ailleurs (Peixoto, 2002a), si les trois premières années de la dernière décennie du $\mathrm{XX}^{\mathrm{e}}$ siècle mettent en relief une tendance, déjà visible entre les années 1970 et les années 1980 (une tendance de ralentissement du rythme d'inscriptions des biens sur la liste du patrimoine mondial), le reste de la décennie inverse très nettement cette tendance. Depuis 1993 le nombre de biens inscrits chaque année sur la liste de l'Unesco a toujours dépassé la moyenne des années 1970 et 1980 , ce qui peut être considéré comme un premier indicateur de l'intensification de la course au statut de patrimoine mondial (Peixoto, 2002a). Cette course, parce qu'elle a acquis, notamment au Portugal et en Europe, les contours d'une compétition entre concurrents professionnels, révèle que le statut de patrimoine mondial fait l'objet du développement de véritables politiques publiques de patrimonialisation, stimulées par la quête d'un label. Il s'agit, certes, d'une course dont la configuration a beaucoup changé dernièrement, soit en raison de la participation et de l'engagement croissants des concurrents, soit en raison de l'intermédiation de l'Unesco.

Pourtant nous savons bien que les dépenses économiques associées au maintien des villes historiques et des ensembles patrimoniaux en général sont assez élevées (Icomos, 1993, pp. 21-22; Patin, 1997, pp. 130-138). Or, dans des communautés qui sont encore loin d'assurer une satisfaction convenable des nécessités premières, le fait de consacrer des ressources à la conservation du patrimoine est souvent 
considéré comme un luxe. Comment expliquer alors l'intensification de la patrimonialisation et, plus concrètement, la course au statut de patrimoine mondial? Qui sont les acteurs de cette course et quelles sont leurs attentes? L'encadrement et l'explication de ce que l'on pourrait appeler la " fièvre de la patrimonialisation ", ou "l'hystérie du patrimoine " (Peixoto, 2002b), nous conduit à centrer la question sur les politiques locales urbaines. En fait, l'intensification de la patrimonialisation résulte, dans une large mesure, de la nécessité de réagir localement à des processus qui sont structurels, au plan économique, et mondiaux en matière d'échelle (Newby, 1994). Nous voulons dire par là que les transformations au sein des structures de l'économie mondiale, caractérisées par la crise des sociétés industrielles, par la croissance de la consommation et par la généralisation des loisirs, ont contraint de nombreuses villes dominées par des économies industrielles ou agricoles à se reconvertir. Les éléments disponibles pour démarrer cette reconversion (surtout au niveau des petites villes et des villes moyennes, dominantes dans le cas portugais) étaient la culture, le passé et le patrimoine (Fortuna, 1997b). Ainsi, un deuxième indicateur, qui permet de mieux caractériser la course au statut de patrimoine mondial, et qui se présente comme une conséquence des transformations que nous venons de repérer, sera-t-il discuté ci-dessous. Il tient au fait que la dernière décennie du millénaire a vu la course être dominée par les villes des pays de l'Europe occidentale (les portugaises inclues, y voulant gagner leur place), lesquelles, curieusement, font partie des principaux marchés d'émission et de réception des flux touristiques.

Ce contexte, et l'objectif de l'Unesco de vouloir freiner le rythme de la patrimonialisation, ont paradoxalement stimulé la course au statut de patrimoine mondial et cela est bien visible dans le cas des politiques patrimoniales portugaises. En instituant un mécanisme de présélection nationale, et des règles plus exigeantes, l'Unesco a rendu la course plus compétitive et conflictuelle ${ }^{3}$. Au Portugal, l'application du mécanisme de présélection a fait apparaître, dans sa première année de fonctionnement, des symptômes de conflictualité. L'État portugais a fini par sélectionner, en vue de présenter une candidature en 2002, la proposition d'inscription des vignes de l'île du Pico aux Açores, rejetant les candidatures de la ville de Marvão et du centre historique et des marais de Santarém, une ville située au nord de Lisbonne dans l'intérieur. Cette sélection s'est faite dans un contexte très particulier, étant donné qu'en 2000 l'État portugais avait soumis au Comité du patrimoine mondial la proposition d'inscription du centre historique de Santarém, inclus dans la liste préalable depuis 1996. L'Unesco, se basant sur le rapport du Conseil International des Monuments et Sites (Icomos), a fait savoir à l'État portugais que la candidature ne serait pas sélectionnée, étant donné que le pays avait déjà inscrit sur la liste du patrimoine mondial plusieurs exemplaires d'architecture gothique (Monastère des Hiéronymites, Monastère de Batalha, Monastère d'Alcobaça et le Couvent du Christ à Tomar). L'État portugais a retiré la candidature de Santarém et, dans un climat de conflit ouvert, a été accusé publiquement par le maire de cette ville de ne pas avoir accompli le travail diplomatique, nécessaire auprès de l'Unesco. Par là même, il a été rendu responsable de l'obligation de suspension de cette candidature. La ville qui, sur le marché touristique, assure sa promotion comme la capitale du gothique au Portugal, a, néanmoins, réitéré sa candidature, en proposant pour inscription son centre historique et les marais environnants. Ce faisant, cette candidature 
vise une classification dans la catégorie des paysages culturels et, de ce fait, augmente les possibilités d'inscription, puisqu'il s'agit d'une catégorie sous-représentée dans la liste (environ trois dizaines), par opposition à la catégorie des biens culturels (environ 6 centaines) visée dans la première tentative de classement. Le fait que l'État portugais n'ait pas présélectionné la candidature de Santarém pour 2002 a été à l'origine, en fonction des antécédents, de la première source de conflit ouvert découlant des mesures adoptées par l'Unesco. En outre, la reformulation de la candidature, dans le but de proposer linscription dans une catégorie sous-représentée, accentue une autre dimension du côté compétitif de la course au statut de patrimoine mondial. Cela veut dire que les candidatures devront veiller à présenter, dans les moindres détails, les critères spécifiques qui augmentent les possibilités d'inscription, selon les caractéristiques que valorise, à chaque instant, la compétition. En ce sens, elles risquent de se convertir en candidatures élaborées au gré de la compétition en cours, privilégiant d'abord les spécificités définies par les critères qui accroissent les possibilités de sélection, plutôt que les caractéristiques intrinsèques du bien qui donne corps à la candidature.

\section{Patrimoine mondial et marketing urbain}

Les villes sont aujourd'hui sujettes à un processus d'évaluation et de hiérarchisation. Cette compétition globale entre espaces conduit les villes à adopter des styles de gestion en vigueur dans les entreprises, accroît la pression pour la médiatisation des politiques urbaines et renforce la tendance au fait que les campagnes de marketing ne se laissent plus circonscrire dans le secteur lucratif (Peixoto, 2000).
Nous pouvons remarquer, dans le domaine des politiques locales patrimoniales, une tendance des villes à s'afficher, sinon à se mettre en scène. Ce qui semble être en cause, c'est un besoin de singularisation, d'affirmation de spécificités permettant aux villes de se positionner dans le jeu de la compétition symbolique, au plan national et international.

Au niveau des politiques urbaines, les particularités locales sont de plus en plus envisagées comme des avantages compétitifs et des ressources au sens économique. Dans les villes qui ont des problèmes d'érosion de leur tissu économique et de perte de compétitivité, le genius loci se présente comme un atout de plus en plus mobilisable dans le cadre des actions dont les objectifs politiques et économiques sont prioritaires. C'est dans cette dimension des politiques locales que l'on trouve les bases de l'intensification des processus de patrimonialisation. La pression de la médiatisation à l'égard des politiques urbaines stimule la production symbolique et la production des espaces emblématiques, ce qui augmente la valeur stratégique du patrimoine (Fortuna, 1997a; Peixoto, 2000). Celui-ci, converti en métalangage des politiques urbaines, est devenu un mot-clé de la rhétorique urbaine et des discours performatifs des acteurs locaux.

Si nous concevons, comme cela semble être le cas des politiques de marketing urbain, la ville comme un objet qui peut s'auto-représenter en tant que produit ciblé par des consommateurs - qu'il s'agisse d'individus à la recherche d'un lieu plus agréable pour habiter, d'un meilleur endroit pour travailler, pour développer une affaire, pour organiser un congrès ou pour passer des vacances - il faut admettre alors que l'image de la ville conditionne fortement sa demande (Kotler et alii., 1993).

De nos jours, beaucoup de villes sont conçues comme un produit et tendent à faire 
une gestion stratégique de leurs images. Dans ce contexte, les politiques de marketing urbain appréhendent les usagers de la ville avec la même logique de la publicité qui considère les individus comme autant de consommateurs qu'il faut persuader. Cela explique, dans une large mesure, la course au statut de patrimoine mondial. Mais explique aussi le fait que presque toutes les villes portugaises, en s'attachant surtout aux produits locaux et régionaux, cherchent à se donner un titre de capitale, tel que "capitale du vin et de la vigne", " capitale du fromage Serra da Estrela ", " capitale de la cerise ", etc. Ce faisant, elles rendent évident le fait que les politiques de gestion des images, en tant qu'élément des politiques patrimoniales et des opérations de réhabilitation urbaine, restent prisonnières d'un phénomène d'invention de slogans.

On peut émettre un doute à propos de l'idée que les villes soient insérées dans un jeu concurrentiel sans précédent. Mais on ne peut douter qu'elles soient devenues un objet dont l'image est constamment travaillée et promue. Même si l'on peut croire légitimement que les villes, qui ne se promeuvent pas et qui ne se publicisent pas délibérément, ne courent pas forcément un risque de stagnation et de déclin, il est indéniable qu'aujourd'hui les lieux sont constamment en train d'être ordonnés, classifiés et évalués dans toutes les dimensions possibles et imaginaires. Cet effet de hiérarchisation (qui est en vogue) conduit les responsables locaux à chercher des occasions pour placer les villes au cœur de la scène médiatique. Dans la rhétorique du jeu concurrentiel intervilles, une ville qui n'est pas médiatique est une ville qui n'existe pas. C'est dans ce contexte que le patrimoine mondial, fonctionnant comme une image de marque globale, est devenu un des principaux labels du marketing. Le bien classé, le centre historique ou un monument, se présente comme une métonymie de la ville même. Par des procédés stylistiques et rhétoriques, on exprime le tout par la partie et les qualités du bien classé sont instrumentalisées pour promouvoir toute la ville.

\section{Patrimoine, tourisme et marché urbain de loisirs}

Même si nous ne développons pas ici l'étroite liaison entre patrimoine mondial et tourisme international, il faut, quand même, souligner qu'au Portugal les investissements dans des projets de patrimonialisation, parfois difficiles à justifier, sont souvent légitimés par la potentielle augmentation des flux du tourisme international (Peixoto, 2002a). Grosso modo, le statut de ville patrimoine mondial est devenu une cible des stratégies des régions où se localisent les principales destinations du tourisme historique et patrimonial. Le changement d'identité symbolique, provoqué par l'obtention de ce statut, fonctionne comme une bannière agitée afin de jouer le rôle d'image de marque des lieux qui essaient de devenir plus compétitifs, surtout au niveau de la captation des flux du tourisme historique et patrimonial.

Depuis les années 1980, les villes ont été redécouvertes à des fins touristiques (Dietvorst, 1994). Même si le tourisme urbain n'a pas connu la massification qui a contaminé le marché touristique depuis les années 1960, la ville est, actuellement (au même niveau que le littoral et la campagne, et avant la montagne) une des quatre grandes destinations de vacances des Européens. Dans le domaine des séjours de courte durée (moins de quatre nuits), les villes constituent la principale catégorie de destinations touristiques à l'échelle européenne, concentrant $50 \%$ de la demande (Merlin, 2001, p. 197). En même temps, dans la dernière décennie du $\mathrm{XX}^{e}$ siècle, la demande 
et la consommation de patrimoine ont connu une croissance inattendue. A cette croissance s'ajoute une remarquable augmentation de l'offre, mise en marche par la multiplication des villes historiques qui cherchent à satisfaire cette offre (Ashworth, Tunbridge, 2000). La consolidation d'un marché urbain de loisirs et l'expansion ainsi que la segmentation de l'industrie touristique sont deux phénomènes liés à cette réalité (Fortuna, 1999). L'un et l'autre se présentent comme des opportunités qui laissent imaginer et qui annoncent que les villes historiques ont devant elles un " brillant passé ". En suivant Lowenthal (1989), nous pourrions conclure que, à l'heure actuelle, rien ne semble se vendre si bien que le passé. Au fur et à mesure que les villes s'affirment comme destinations touristiques, le patrimoine devient une grande industrie internationale. L'action développée par l'Unesco dans le domaine de l'identification de sites naturels et des biens culturels, ainsi que son inscription ultérieure sur la liste du patrimoine mondial, a beaucoup contribué à soutenir cette industrie, peut-être pas intentionnellement, mais en tout cas de façon effective (Carr, 1994).

Pour revenir à notre argument selon lequel la patrimonialisation est un phénomène indissociable de la consolidation d'un marché urbain de loisirs et de l'expansion et de la segmentation de l'industrie touristique, on doit constater que la diffusion de nouvelles images qui nourrit ce marché, s'effectue principalement de deux façons: soit à travers la dynamisation culturelle, telle que l'organisation des événements culturels, et de l'inscription de symboles modernisateurs dans les paysages urbains, soit à travers l'instrumentalisation, la réinvention et la revalorisation du patrimoine historique, qui devient une ressource symbolique de stratégies visant à convertir la marque de tradition de ce patrimoine en capital d'innovation (Fortuna, 1997a). Les villes sont de plus en plus engagées sur des projets qui essaient d'offrir une culture urbaine axée sur la jouissance de l'espace public. Au niveau des politiques urbaines, les centres historiques et l'espace public sont envisagés comme des équipements culturels. Dans le contexte du marché urbain de loisirs et du tourisme, les centres historiques sont en train de devenir des scènes de sociabilité et de mise en scène de la vie quotidienne, tout en se constituant comme une sorte de nouvelle réalité allégorique des villes, qui se manifeste de trois façons. D'un côté, les centres historiques sont de plus en plus présentés comme l'expression concrète d'une idée d'espace public. Ainsi, sous la forme figurée de l'imbrication entre consommation et loisir, les centres historiques sont une allégorie de cet espace public apparemment perdu et qu'il faudrait récupérer. D'un autre côté, l'animation croissante des centres historiques et leur " touristifisation ", constituent et font naître des expressions performatives et plastiques qui visent à suggérer des idéaux de citoyenneté et de participation civique. Finalement, les centres historiques fonctionnent comme allégorie pour les situations dans lesquelles leur splendeur et la qualité urbanistique de leurs espaces, souvent dissonantes du reste de la ville environnante, les transforment en une sorte d'œuvre d'art qui représente une idée abstraite de qualité de vie. Dans ce sens, ils fonctionnent en tant qu'image métonymique de la ville, invitant à prendre la partie, ordonnée et embellie, pour le tout.

Ainsi, l'intensification des processus de patrimonialisation est liée aussi au fait que le patrimoine est une ressource importante de ce qu'on pourrait appeler le marché urbain des loisirs. Limbrication croissante entre consommation et loisir, d'une part, et l'encadrement de ces dynamiques dans l'espace, d'autre part, a conduit les politiques locales à s'occuper 
plus activement des centres urbains et de leurs fonctions culturelles. Quand nous parlons aujourd'hui de la compétition intervilles, nous parlons, en même temps, des actes de consommation, même s'il ne s'agit que des actes de consommation visuelle, et des moments de contact avec l'espace vécu de la ville (Fortuna, 1997b). Ce que les villes essayent de faire aujourd'hui, c'est de spatialiser ces deux dynamiques (deux dynamiques fort imbriquées façonnant le quotidien des villes) dans un cadre patrimonial qui aide, à travers la stylisation, à travers le traitement plastique de l'espace, et à travers l'offre d'une marque de tradition, à promouvoir la fréquentation de l'espace public.

Coimbra, la vieille ville universitaire, peut être retenue comme exemple d'une des villes portugaises qui nous permettent de rendre compte des quelques dimensions de ce marché urbain des loisirs et du tourisme. Nous assistons à Coimbra, depuis une quinzaine d'années, au développement de formes variées d'esthétisation et de stylisation de l'espace urbain ainsi qu'à l'essor d'une économie symbolique du patrimoine (Zukin, 1997) ${ }^{4}$. Parmi les formes d'esthétisation, peuvent plus particulièrement retenir notre attention, la réactivation des coutumes passées, voire la fabrication et l'exposition des produits artisanaux et gastronomiques. Il s'agit, dans les deux cas, de rassembler des éléments culturels et historiques, constitutifs d'un patrimoine susceptible d'être publicisé comme singulier. Mis en scène et exposés à travers des événements combinant le traditionnel et le festif, ces aspects acquièrent le statut d'un signe et d'une image destinés à être "achetés " par les touristes.

La façon dont ce processus se concrétise met en évidence une certaine distanciation et quelques effets pervers, une distanciation, notamment, entre les pratiques quoti- diennes de l'espace vécu et les actions qui visent la concentration des éléments culturels nécessaires à la mise en scène et à l'animation d'un espace investi d'une forte valeur représentationnelle. Le " marché aux oignons ", réalisé pendant une semaine du mois d'août dans une place centrale du centre historique, exemplifie le phénomène de traditions réifiées pour nourrir le marché urbain du tourisme et des loisirs. Cet événement a été " repris " par le groupe folklorique Os camponeses (les paysans) qui, soutenu par les autorités locales, a récupéré une tradition lointaine. Plus qu'un marché pour vendre des oignons, l'événement se concentre dans l'animation de la place, à travers l'exécution de musiques et danses populaires, la promotion des produits gastronomiques, eux aussi hérités de traditions passées. Si le slogan de l'une des dernières éditions entendait promouvoir les touristes comme principaux acteurs de l'existence de ce marché séculaire (en postulant " nous voulons voir les touristes partant avec les oignons sous le bras"), le fait est cependant que, d'après les témoignages des petits producteurs venus vendre leur production, les touristes ne se sont montrés intéressés qu'à prendre des photos pour saisir l'altérité exotique de cette autre Coimbra.

La question qui se pose ici est de savoir ce que représente cet excès d'animation. Nous pouvons suggérer déjà qu'il résulte de la quête de légitimation par les groupes qui promeuvent les activités d'animation dans un marché saturé par des offres semblables. Mais cette animation, souvent exacerbée, représente beaucoup plus que cela. Elle est aussi, du point de vue des responsables des politiques locales qui soutiennent ces groupes, une réponse à l'état de dépression qui se manifeste dans des centres historiques, habités de plus en plus par une popu- 
lation âgée, incapables aussi de générer leurs propres dynamiques. Contre la dévitalisation, et parfois même contre la vitalité qui existe dans les centres historiques, nous assistons, à travers ce type de manifestations festives et d'actions de réhabilitation urbaine, à l'émergence d'expressions performatives, artistiques et techniques entendant diffuser des idéaux de citoyenneté et de participation civique. Dans ce contexte, l'espace animé se présente comme producteur des signifiés sociaux, comme si le visuel était une condition fondatrice et suffisante de l'émergence de nouvelles sociabilités.

Mais, à Coimbra les politiques de réhabilitation urbaine ont, elles aussi, créé des conditions pour la consolidation d'un marché urbain du tourisme et des loisirs. Ainsi, un espace central a été aménagé pour favoriser la concentration d'éléments patrimoniaux. Ici, une panoplie d'objets patrimoniaux se résume à des bâtiments réhabilités pour façonner un décor. Nous sommes alors devant un espace scénique de jouissance esthétique censée représenter l'esprit du lieu. Nous repérons dans l'axe piétonnier qui s'étend d'Arco de Almedina au Largo da Sé Velha, en passant par la rue Quebra Costas, l'essor de l'économie symbolique du patrimoine. En traversant cet axe, incontournable pour les touristes qui visitent l'université et le centre historique, nous sommes confrontés à Arco de Almedina, avec un petit établissement vendant de vieux livres profusément exposés dans la rue elle-même. En symbolisant, dès lors, la vétusté de la ville, cet établissement reproduit incessamment la chanson typique de Coimbra. Nous pouvons y trouver, sous la forme des cartes postales et des faïences, les symboles les plus stéréotypés du Portugal et de Coimbra: les images des anciens bateaux des découvertes portugaises, des quartiers typiques de Lis- bonne, des palais de Sintra, du coq de Barcelos, de la tour de l'université et des étudiants, habillés selon la tradition. En montant, jusqu'à Largo da Sé Velha, nous trouvons un nombre croissant de maisons d'artisanat. Elles se sont installées dans les quinze dernières années et commercialisent presque exclusivement des céramiques et des faïences " traditionnelles " de Coimbra.

C'est là que les touristes, avides de découvrir la ville, peuvent finalement trouver les objets " traditionnels et uniques " permettant de " témoigner " de leur passage par Coimbra. Beaucoup de ces souvenirs sont élaborés pour sembler anciens. La céramique, notamment les carrés d'azulejos, cherche à imiter la production portugaise de l'époque baroque, représentée dans les monuments de la ville.

Dans le contexte des politiques locales, l'importance des processus de patrimonialisation ne réside pas vraiment dans les stratégies de conservation des signes architectoniques du passé, mais plutôt dans l'encouragement qu'ils peuvent donner à la création culturelle et aux actes de dynamisation et d'animation de l'espace public. Le patrimoine fait l'objet de politiques publiques et, pour les élus locaux, il faut absolument l'inscrire dans les dynamiques économiques et sociales de l'espace urbain, car le patrimoine est un élément de modernisation et c'est justement dans la tension entre patrimoine et transformation de l'espace que s'ouvrent les portes de la modernité (Poulot, 1998).

Les processus de patrimonialisation, soit parce qu'ils résultent de la pression de secteurs de la population locale, intéressés à renforcer leurs droits civiques, soit parce qu'ils résultent de logiques de rivalité entre lieux qui visent à maintenir ou à augmenter leur prestige, sont souvent de nature réactive 
et finissent fréquemment par se convertir en une fin en soi. La course au statut de patrimoine mondial et l'animation croissante des centres urbains sont des conséquences de cet effet de reproduction en série d'un modèle de développement urbain qui, aux yeux des responsables locaux, crée de nouvelles occasions d'affirmation des villes, de nouveaux avantages dans le domaine touristique et commercial et de nouveaux éléments qui aident à renforcer l'identité locale. La conséquence la plus évidente de cette tendance est que, dans les années 1990, le nombre de villes historiques qui sont en compétition pour capter le même marché touristique n'a cessé d'augmenter.

Au Portugal nous pouvons retenir plusieurs exemples d'importation des modèles de gestion mondialisés qui, en même temps, font ressortir que le patrimoine fait l'objet de politiques publiques et que ces politiques sont au service des opérations hygiéniques dont le but est d'aménager l'espace pour le marché urbain du tourisme et des loisirs. A Guimarães (petite ville au nord, " berceau " de la nationalité portugaise), par exemple, le maire a voulu installer dans le centre historique un système de vidéosurveillance. Cette mesure avait un caractère expérimental puisqu'il s'agissait d'assurer une protection supplémentaire aux monuments historiques et aux biens patrimoniaux du centre urbain. Nous étions en 1997 et Guimarães était fortement engagée dans la candidature au statut de patrimoine mondial, reconnaissance qu'elle obtiendra en 2001.

Force est de constater que, dans le centre historique de Guimarãe, les modes traditionnels de gestion des risques urbains sont encore dominants. Ils sont basés dans l'urbanisme de proximité, dans les relations de voisinage, dans l'interconnaissance, dans un certain esprit de collectivité et dans l'existence d'institutions de proximité, capables d'intervenir dans les domaines où les risques se manifestent. Mais le centre historique est de plus en plus habité par des individus tendanciellement âgés et cela vient mettre en cause les modes traditionnels de gestion des risques. A cela nous pourrions ajouter l'effet de développement des nouvelles dynamiques au centre historique, notamment les activités nocturnes de loisir. Cette " nouvelle " fonction, visible dans les places centrales (Place de S. Tiago et Place da Oliveira), entraîne, elle aussi, des nouveaux risques.

Pour répondre à l'émergence des nouveaux risques, l'option de la vidéosurveillance s'est présentée, aux yeux des responsables locaux, comme la solution la plus évidente. Dans des espaces labyrinthiques et denses, comme le centre historique de Guimarães, où des nouveaux risques émergent, la vidéosurveillance est souvent envisagée comme l'équivalent fonctionnel moderne du panopticum de Bentham. La rigidité de l'espace est ainsi contournée par la plasticité d'une technologie qui permet de contrôler sans être remarquée. Toutefois, à Guimarães la solution a été refusée, non seulement parce qu'elle n'était pas encadrée dans la loi, mais parce qu'elle répondait plus aux aménagements nécessaires pour le développement d'un marché urbain du tourisme et de loisirs qu'aux exigences de la population locale. En fait, nous étions devant une option politique qui, pour une majorité de l'assemblée municipale, essayait d'amplifier et d'exacerber le sentiment d'insécurité afin de permettre l'installation d'un système de contrôle qui augmente effectivement le risque d'insécurité.

Ces modèles mondialisés, conçus pour aménager les centres historiques afin d'y faciliter le développement des pratiques touristiques et de loisir, en diffusant l'idée qu'une société régulée et formatée convient mieux à tout le monde, sont souvent, comme cela a été 
le cas à Guimarães, contestés et refusés. Dans ce cas, les nouveaux risques, associés à la solution de la vidéosurveillance, s'avèrent une intromission dans la vie privée des citoyens, par le biais de possibles diffusions des images captées, du fait que ces dernières puissent être une source de réactions immédiates et primaires de la part des surveillants vis-à-vis des utilisateurs de l'espace public.

\section{La " soutenabilité " culturelle des politiques patrimoniales locales et des opérations de réhabilitation urbaine}

Le fait que les politiques patrimoniales et les opérations de réhabilitation urbaine au Portugal soient marquées par une course au titre de patrimoine mondial, par l'importation des modèles mondialisés de gestion urbaine, et par des formes exacerbées d'animation et d'esthétisation de l'espace nous conduit à poser la question de la "soutenabilité " culturelle de ces politiques et des centres urbains où elles sont adoptées.

Prenons encore un exemple issu de la réalité portugaise. En novembre 2001, la mairie de Porto, la deuxième plus grande des villes portugaises, a mis en marche, à travers l'Agence de Modernisation de Porto, une expérience pilote ciblant la valorisation du paysage urbain. Il s'agissait d'entreprendre un programme d'amélioration de l'image de l'espace public, orienté vers la réhabilitation des façades d'édifices publics et privés, vers le renouvellement du mobilier urbain et vers la préservation des objets d'art public. Le premier de ces objectifs a été le plus important et c'est celui auquel nous accorderons ici le plus d'attention'.

Dans les deux premiers mois de fonctionnement, ont été approuvées dans le cadre du programme de réhabilitation de façades six dizaines de candidatures. Ce programme nous intéresse parce qu'il traduit un évident caractère expérimental, dans la mesure où il cherche à concrétiser les opérations de réhabilitation en instituant des partenariats avec des entreprises privées. Selon les termes du partenariat, les entreprises prenant part aux coûts, ont le droit, à titre compensatoire, d'afficher des toiles publicitaires devant les façades en réhabilitation, sans devoir payer les taxes municipales.

Du point de vue institutionnel, ce projet se présente comme une opportunité pour renforcer « les éléments paysagers qui caractérisent l'image de la ville de Porto, tout en stimulant les valeurs civiques qui rendent les relations de citoyenneté plus fortes, équitables, riches et socialement équilibrées ». Le programme de valorisation du paysage urbain " Porto com pinta " parie sur sa capacité de diffuser une culture de préservation et de réhabilitation du patrimoine édifié qui sera, par elle même, capable de faire émerger des sentiments d'identification à l'égard de la ville. Dans ce sens, même s'il s'agit d'édifices privés, les façades sélectionnées prioritairement sont celles qui ont une valeur emblématique pour la ville et qui, jouant le rôle de signe identitaire, sont envisagées comme un bien public.

Cette philosophie du "Porto com Pinta", qui investit les opérations de réhabilitation d'un pouvoir de création de comportements et liens de citoyenneté, apparaît comme le leitmotiv des politiques patrimoniales locales et nourrit les modèles mondialisés de transformation de l'espace urbain.

Or, le tourisme est couramment envisagé comme une façon d'obtenir la reconnaissance politique dans un monde compétitif (Robinson, 2001). De la même façon, le statut de patrimoine mondial, soit pour sa 
valeur intrinsèque et symbolique, soit pour sa valeur fonctionnelle dans le domaine de la projection médiatique des villes et de l'animation urbaine, constitue une source de légitimation politique nationale et locale. Le tourisme, le statut de patrimoine mondial et les opérations de réhabilitation se combinent, tant au niveau national qu'au niveau local, pour augmenter le prestige et l'estime de soi des entités propriétaires des biens visités et classés. Paradoxalement, les États et les villes qui cherchent à affirmer leur compétitivité et leur modernisation semblent mieux atteindre leurs objectifs quand ils réussissent à façonner et à médiatiser des témoins de leur vétusté. Le statut de patrimoine mondial, en faisant preuve d'un passé insigne et unique, est un instrument privilégié de cette logistique symbolique qui aide à atteindre la reconnaissance politique dans un monde compétitif. Le tourisme, de son côté, en mettant en évidence cette compétitivité et, de ce fait, la modernité du pays, est une mesure de confirmation de la valeur culturelle des biens visités. Mesure qui est d'autant moins controversée que la reconnaissance est, en général, octroyée par des étrangers. Toutefois, cette légitimation politique est loin d'empêcher les usages très diversifiés du statut de patrimoine mondial et de permettre que le tourisme acquière une légitimité incontestable.

Le statut de l'Unesco devient un des éléments centraux de la rhétorique et de la lutte politiques sous-jacentes aux projets de réhabilitation urbaine. Ce statut est fréquemment évoqué pour légitimer ou pour critiquer certaines stratégies d'intervention. Il est cité pour contester ou justifier le statu quo. En fait, en devenant une distinction et la référence symbolique et emblématique par excellence des processus de patrimonialisation, le statut de patrimoine mondial semble avoir acquis une aura qui permet d'atteindre un niveau inimaginable de consensus en ce qui concerne les choix au niveau des politiques urbaines.

Le recours au patrimoine afin d'assurer la promotion nationale et internationale de la ville, fait que beaucoup d'élus locaux investissent particulièrement au niveau de la construction d'un discours, d'une iconographie et d'un esthétisme de la ville, dont le statut de patrimoine mondial devient une référence emblématique. Mais, souvent, ces images urbaines sont forgées au travers des protocoles de presse, ayant pour base des techniques de marketing et une forte dimension rhétorique, et non pas, ce qui devrait être le cas, un travail de transformation de l'espace urbain et de son paysage social. En fait, la quête du statut de patrimoine mondial peut être subordonnée à une stratégie de construction d'une image de marque qui n'a pas une correspondance au niveau des dynamiques de l'espace urbain. Cela se produit, comme l'affirme Bourdin (1996), soit quand se développe un patrimoine sans projet, soit quand le patrimoine devient son propre projet. Même en croyant aux pouvoirs magiques du tourisme et du patrimoine, nous devrions nous poser la question de savoir si les opérations de réhabilitation doivent les privilégier à tout prix. Il n'est pas rare que l'anoblissement du passé ait pour conséquence l'oubli et l'appauvrissement du présent, et que le prix de la reconnaissance extérieure soit, en même temps, l'effacement des caractéristiques locales. Dans ce sens, et considérant par exemple les réactions populaires à l'égard de la rigidité que la patrimonialisation impose, nous devons nous interroger afin de savoir si les habitants se reconnaissent dans une identité urbaine qui leur est imposée, dans la mesure où les initiatives 
patrimoniales, visant à promouvoir la visibilité et la compétitivité de la ville, sont de l'ordre du prestige et de la performance (Bourdin, 1996; Legué-Dupont, 1996).

La "fièvre de la patrimonialisation", que nous constatons au Portugal, matérialisée par la quête du statut de patrimoine mondial, n'a pour seul but que de fournir aux villes le statut de " marchandise patrimoniale " (Bourdin, 1996). Les deux principaux problèmes de cette fureur patrimoniale tiennent, d'un côté, à l'action des autorités municipales qui ont du mal à mettre en place une politique de gestion. Ils essaient d'obtenir une médaille, plutôt que de développer une politique cohérente du patrimoine. D'un autre côté, ces mêmes autorités prétendent faire, dans le cadre des candidatures au patrimoine mondial, ce qui aurait dû être fait, depuis longtemps, dans le domaine de la protection du patrimoine.

\section{2}

Ce texte part de la thèse qu'on est en face d'une course au statut de patrimoine mondial et que cette course façonne les politiques patrimoniales locales portugaises. Cette course fait, ainsi, l'objet d'une analyse dans le contexte de la promotion du marché urbain de loisir et de tourisme, tout en cherchant à caractériser les diverses dimensions de cette course ainsi que les complicités et les tensions entre patrimoine, villes et tourisme. Globalement, nous pourrions retirer de l'analyse effectuée cinq conclusions générales.

La première conclusion tient au fait que, dans les années 1990, nous assistons à une intensification sans précédent de cette course. Ce qui l'explique, dès lors, ce sont les changements structurels au niveau de l'économie mondiale et leurs impacts au niveau local. L'expansion de l'activité touristique, la conso- lidation d'un marché urbain de loisirs et l'importance croissante des politiques locales urbaines sont des facteurs qui encadrent et justifient cette course. D'autre part, cette course se développe dans un contexte caractérisé par une territorialisation croissante de la compétitivité, en ce sens que les spécificités locales sont devenues une cible de grands investissements symboliques, ainsi que par une nette et profonde pression pour la médiatisation des politiques locales.

La deuxième conclusion nous renvoie au fait que le statut de patrimoine mondial est devenu une cible stratégique des villes et des pays où sont localisés les principaux marchés du tourisme historique et patrimonial. Ce statut semble être convoité dans le but de fonctionner comme image de marque de la compétitivité. L'accroissement de la demande dans ce secteur du marché touristique s'est accompagné d'une remarquable augmentation de l'offre, ce qui oblige à la création et à l'invention de distinctions symboliques possibles et imaginaires. Dans ce sens, le statut de l'Unesco a élevé, de façon évidente, l'expansion du patrimoine en tant qu'industrie internationale.

Une troisième conclusion rend compte de la contradiction qu'il semble y avoir entre la philosophie sous-jacente à la Convention de l'Unesco de 1972 et les résultats pratiques de son application, ainsi qu'en ce qui concerne les mesures adoptées pour éviter la banalisation du statut de patrimoine mondial. Ces mesures conduisent, à notre avis, à la densification compétitive et conflictuelle de la course. A la limite, nous pouvons affirmer que le patrimoine mondial, à l'opposé de la matrice doctrinale qui l'a fait émerger, est en train de fonctionner plutôt comme une cause de conflit national et local que comme instrument de réconciliation mondiale.

Comme quatrième conclusion, nous rete- 
nons l'idée que, dans le contexte du marché urbain de loisirs et du tourisme, les centres historiques se convertissent, par l'application des politiques patrimoniales, en scènes de sociabilité " spectacularisées " et de mise en scène de la vie quotidienne, constituant ainsi une sorte de nouvelle réalité allégorique des villes.

Comme cinquième et dernière conclusion, nous voudrions souligner que le tourisme et le statut de patrimoine mondial, dans l'étroite relation qui les unifie, agissent en tant que sources de légitimation politique nationale et locale, augmentant le prestige et l'auto- estime des États et des villes. La légitimation qu'ils offrent ne cache pas les conduites arbitraires liées, soit au tourisme, soit au patrimoine. S'il est vrai que le patrimoine est conservé parce qu'il constitue une ressource qui nourrit le marché touristique, il ne faut pas oublier qu'une très grande quantité de ce qu'on appelle patrimoine a été détruit à cause de ce même tourisme. D'un autre côté, les discours sur le patrimoine et sa valorisation correspondante dans le contexte du marché touristique, oscillent entre, d'une part, une utilisation pensée et stratégique des ressources patrimoniales tendant à promouvoir le développement urbain et, d'autre part, une rhétorique vidée de sens qui, très souvent, n'est autre chose que des protocoles médiatiques.

\section{$\underset{\text { Notes }}{\approx}$}

1. Nous employons ici le terme "soutenabilité ", utilisé dans les langues portugaise, espagnole, anglaise et même française, parce que ce néologisme apparaît de plus en plus lié aux questions du développement durable et de l'équilibre des systèmes en signifiant le maintien en valeur de l'ensemble des patrimoines.

2. Nous avons pu repérer dans la presse l'intention de promotion des candidatures suivantes: Algar do Carvão (Aux Açores), Almeida, Aqueduc das Águas Livres (à Lisbonne), Baixa pombalina (à Lisbonne), Barcelos, Castro do Cadaval, Caves du porto (a Gaia), Centre historique de Coimbra, Funiculaire do Bom Jesus de Braga, Furna do Enxofre (aux Açores), Marvão, Miranda do Douro, Obidos, Sagres, Santarém, Valença.

3. L'Unesco, en définissant des mécanismes de restriction, a engagé plus fortement les États nationaux dans le processus de certification. Le mécanisme de présélection nationale, attestant les capacités pour entrer en compétition à un niveau supérieur, fait ressortir le besoin de garantir en temps et lieu les conditions de succès: le temps nécessaire pour la bonne préparation de la candidature, le besoin de connaître et d'évaluer de façon permanente les concurrents directs au niveau national, la professionnalisation au niveau de la préparation de la proposition et, entre autres, la capacité d'exercer l'influence indispensable à la présélection.

4. Une analyse élargie et détaillée de ce cas peut être consultée à partir de Frias e Peixoto (2002).

5. D'ailleurs, le nom du programme a surtout à voir avec ce premier objectif. "Porto com pinta " ou, en français, Porto avec des marques de peinture, signifie deux choses en même temps: d'un côté, la ville deviendrait plus colorée, de l'autre, l'expression portugaise « ter pinta " signifie avoir bonne mine. 


\section{Références}

Ashworth G., Tunbridge J. (eds.), The Tourist-Historic City - Retrospect and Prospect of Managing the Heritage City, Amsterdam, Pergamon, 2000.

Bourdin A., "Sur quoi fonder les politiques du patrimoine urbain? ", Les Annales de la Recherche urbaine, $\mathrm{n}^{\circ}$ $72,1996$.

Carr E., "Tourism and Heritage. The Pressures and Challenges of the 1990's ", in Ashworth G., Larkham P. (eds.), Building a New Heritage. Tourism, Culture and Identity in the New Europe, London and New York, Routledge, 1994.

Dietvorst A., "Cultural Tourism and Time-Space Behaviour ", in Ashworth G., Larkham P. (eds.), Building a New Heritage. Tourism, Culture and Identity in the new Europe, London and New York, Rourledge, 1994.

Fortuna C. (ed.), Cidade, cultura e globalização, Oeiras, Celta, 1997a.

Fortuna C., "The Shaw Must Go on: Why Are Old Cities Becoming Fashionable?", in Bovone L. (ed.), Mode: produrre cultura, creare comunicazione, Milano, Franco Angeli, 1997b.

Fortuna C., Identidades, percursos, paisagens culturais : estudos sociológicos de cultura urbana, Lisboa, Celta, 1999.

Frias A., Peixoto P., " Representaçāo imaginária da cidade. Processos de racionalização e de estetização do património urbano de Coimbra ", Oficina do Centro de Estudos Sociais, n 183, 2002.

Icomos, Tourism at World Heritage Cultural Sites. The Site Manager's Handbook, Madrid, World Tourism Organization, 1993.

Kotler P., Haider D., Rein I., Marketing Places. Attracting Investement, Industry, and Tourism to Cities, States and Nations, New York, The Free Press, 1993.

Legué-Dupont P., "Les États-Unis: un site exemplaire ", Les Annales de la Recherche Urbaine, no 70,1996.

Lowenthal D., "Nostalgia Tells Like it Wasn't ", in Shaw C., Chase M. (eds.), The Imagined Past. History and Nostalgia, Manchester and New York, Manchester University Press, 1989.

Merlin, P., "Tourisme et aménagement touristique ", Notes et Etudes Documentaires, Paris, La Documentation française, 2001.

Newby P. T., "Tourism. Support or Threat to Heritage? ", in Ashworth G., Larkham P. (eds.), Building a New Heritage. Tourism, Culture and Identity in the New Europe, London and New York, Routledge, 1994.

Patin V., Tourisme et patrimoine en France et en Europe, Paris, La Documentation française, 1997.

Peixoto P., " Gestão estratégica das imagens das cidades: análise de mensagens promocionais e de estratégias de marketing urbano ", Revista Critica de Ciências Sociais, no 56, 2000.

Peixoto P., "A corrida ao status de património mundial e o mercado urbano de lazer e turismo ". Veredas. Revista cientifica de turismo, $\mathrm{n}^{\circ} 1,2002 \mathrm{a}$.

Peixoto, P., "Os meios rurais e a descoberta do património ", Oficina do Centro de Estudos Sociais, no 175, $2002 \mathrm{~b}$.

Poulor D., "Le patrimoine et les aventures de la modernité ", in Poulot D. (dir.), Patrimoine et modernite, Paris, L'Harmattan, 1998.

Robinso, M., "Tourism Encounters: Inter- and Intra-Cultural Conflicts and the World's Largest Industry ", in Alsayyad N. (ed.), Consuming Tradition, Manufacturing Heritage. Global Norms and Urban Forms in the Age of Tourism, London et New York, Routledge, 2001.

Scarce J., "Tourism and Material Culture in Turkey ", in Hitchcock M., Teague K. (eds.), Souvenirs: the Material Culture of Tourism, Aldershot et Burlington, Ashgate, 2000.

Zukin S., The Cultures of Cities, Oxford, Cambridge, Blackwell, 1997. 